\title{
Editorial: Inorganic Chemistry Editor's Pick 2021
}

\author{
Luís D. Carlos ${ }^{1 *}$ \\ ${ }^{1}$ Phantom-g, CICECO - Aveiro Institute of Materials, Department of Physics, University of Aveiro, Aveiro, Portugal
}

Keywords: lanthanides, coordination polymers, MOFs, carbon nanotubes, oxysulfides

Editorial on the Research Topic

Inorganic Chemistry Editor's Pick 2021

We are pleased to introduce the collection Inorganic Chemistry Editor's Pick 2021.

This collection showcases 19 manuscripts (including four review papers) from the past couple of years authored by scientists of distinct countries such as Australia, Chile, China, France, Germany, India, Iran, Italy, Pakistan, Poland, Portugal, Saudi Arabia, Switzerland, and United Kingdom. The collection aims to further support Frontiers' strong community by recognizing highly deserving authors. The selected manuscripts highlight the broad diversity of research performed across the section, including the synthesis and general characterization (e.g., structural, magnetic, optical) of coordination polymers and metal-organic frameworks (MOFs) and its application for sensing, nanoplates for supercapacitors, nanophosphors for upconversion and the dielectric properties of carbon nanotubes, for instance. The review manuscripts encompass the biomolecular modification of MOFs, bulk and nano metal oxysulfides, rhenium complexes as probes for prokaryotic and fungal cells, and atomistic simulations of rare-earth orthophosphates.

All research presented here illustrates strong advances in experiments, theory, and in silico with applications to compelling problems aiming to put a spotlight on the main areas of interest for the section.

Specialty section:

This article was submitted to Inorganic Chemistry,

a section of the journal

Frontiers in Chemistry

Received: 14 April 2021 Accepted: 16 April 2021

Published: 28 April 2021

Citation:

\section{AUTHOR CONTRIBUTIONS}

The author confirms being the sole contributor of this work and has approved it for publication.

Conflict of Interest: The author declares that the research was conducted in the absence of any commercial or financial relationships that could be construed as a potential conflict of interest.

Copyright (c) 2021 Carlos. This is an open-access article distributed under the terms of the Creative Commons Attribution License (CC BY). The use, distribution or reproduction in other forums is permitted, provided the original author(s) and the copyright owner(s) are credited and that the original publication in this journal is cited, in accordance with accepted academic practice. No use, distribution or reproduction is permitted which does not comply with these terms. 